\title{
Long-Term Survival of Over 6 Years with Afatinib Sequential Treatment in a Patient with EGFR Mutation-Positive Non-Small Cell Lung Cancer: A Case Report
}

\author{
Hai-Yan Tu ${ }^{1}$ Y Yang-Si Li ${ }^{1} \cdot$ Jin-Ji Yang ${ }^{1} \cdot$ Hua-Jun Chen ${ }^{1} \cdot$ Ben-Yuan Jiang ${ }^{1} \cdot$ Wen-Zhao Zhong ${ }^{1} \cdot$ Xue-Ning Yang ${ }^{1}$. \\ Yi-Long $\mathrm{Wu}^{1}{ }^{1}$
}

Accepted: 6 March 2021 / Published online: 16 April 2021

(c) The Author(s) 2021

Lung cancer is the leading cause of cancer-related death worldwide [1], with non-small cell lung cancer (NSCLC) accounting for approximately $85 \%$ of all cases [2], and lung adenocarcinoma and squamous cell carcinoma representing the most common NSCLC subtypes [3].

An increased understanding of the genomic landscape in NSCLC has led to the identification of targetable molecular pathways and the subsequent development of highly targeted treatments that demonstrate substantial clinical benefit compared with traditional platinum-based chemotherapy [4]. In particular, identification of epidermal growth factor receptor (EGFR) activation-most commonly resulting from L858R point mutation or deletions within exon 19 (Del19) —as an oncogenic driver in NSCLC, has led to the development and approval of several EGFR tyrosine kinase inhibitors (TKIs) [5]. Among these, afatinib, an ErbB family blocker that irreversibly binds and inhibits the activity of EGFR, HER2 (ErbB2), and HER4 (ErbB4), and blocks transphosphorylation of HER3 (ErbB3) [6], is approved for first-line treatment of NSCLC in patients with non-resistant (sensitizing) EGFR mutations [7, 8].

Unfortunately, acquired resistance is a key challenge in the use of EGFR TKIs, and almost all patients ultimately experience tumor progression despite high initial response rates $[9,10]$. The predominant resistance mechanism, detected in 50-70\% of tumors [11-13], is the secondary EGFR T790M mutation, which appears to result in steric interference of EGFR TKI binding [14], or enhanced EGFR

Yi-Long Wu

syylwu@live.cn

1 Guangdong Lung Cancer Institute, Guangdong Provincial Key Laboratory of Translational Medicine in Lung Cancer, Guangdong Provincial People's Hospital and Guangdong Academy of Medical Sciences, 106 Zhongshan Er Rd, Guangzhou 510080, China affinity for ATP $[15,16]$. Several third-generation EGFR TKIs have been developed to overcome T790M-driven resistance, including osimertinib, an irreversible inhibitor of EGFR-sensitizing and T790M-resistance mutations, with selectivity over wild-type EGFR [17]. However, resistance to osimertinib is also inevitable, occurring through heterogeneous mechanisms, including acquisition of tertiary $E G F R$ mutations or EGFR-independent mechanisms [9].

Given the availability of several EGFR-targeted drugs, there is currently considerable debate regarding optimal treatment sequencing in EGFR mutation-positive NSCLC to maximize long-term clinical benefit, with little direct evidence from comparative trials available to inform these decisions [5, 18, 19]. Herein, we describe the case of a patient with pleural-disseminated EGFR mutation-positive stage IV lung adenocarcinoma, who achieved long-term clinical benefit of more than 6 years with sequential EGFR TKI treatment.

A 38-year-old non-smoking Chinese male, with no family history of tumors, presented with an intermittent cough in June 2009. Computed tomography (CT) scans showed a flaky shadow on the left lower lung; subsequent follow-up 5 months later indicated no change in the lesion and carcinoembryonic antigen (CEA) level was normal ( $\leq 5 \mathrm{ng}$ / $\mathrm{mL}$ ). In November 2010, however, CT scan indicated that the lesion had become denser, and blood CEA level was $110 \mathrm{ng} / \mathrm{mL}$. Subsequent positron emission tomography/ CT scan indicated stage IB lung cancer (cT2aN0M0 [7th edition of tumor, node, metastasis [TNM] classification]). The patient was followed-up until December 2010, when video-assisted thoracic surgery (VATS) was performed. During VATS, bloody pleural effusion $(80 \mathrm{~mL})$ was identified, and multiple widespread nodules were observed in the parietal pleura, pericardium, and diaphragmatic muscle. The parietal nodules, found accidentally and which had not been observed in the CT scan before surgery, were removed 
from the left upper lung for biopsy, via wedge resection, and pathological examination revealed NSCLC adenocarcinoma of the left upper lung. The patient was then classified as having pT4N0M1a (pleura) stage IV disease. Genetic analysis using the amplification-refractory mutation system (ARMS) indicated that the tumor was positive for $E G F R$ Del19 (December 2010; Fig. 1).

The patient received first-line oral afatinib $40 \mathrm{mg} /$ day from late December 2010, as part of the LUX-Lung 6 clinical trial and achieved a partial response as the best overall response 3 months later (Fig. 2). This was associated with a $67 \%$ decrease in target lesion size, as well as the disappearance of pleural effusions, and pleural and pericardial nodules. Notable drug-related adverse reactions were grade 2 erythema, and grade 1 diarrhea, paronychia, and dry skin. The patient remained asymptomatic but developed enlargement of target lesions and recurrence of small pleural effusions and pericardial nodules in December 2013. The initial progression-free survival (PFS) time (PFS1) was 36 months. He then developed cough, tachypnea, and large pleural effusions in the left lung. Post-initial PFS (PFS2) was confirmed as 4 months, for a total PFS of 40 months on first-line afatinib (Fig. 1). Evaluation in April 2014 showed significant pleural thickening in the left side, with a large volume of left pleural effusion that was partially enclosed, and multiple nodular lesions emerging in the right lung and mediastinal lymphadenectasis.

Pleural re-biopsy in April 2014 reconfirmed the presence of adenocarcinoma, which was positive for EGFR Del19 (ARMS method) and negative for T790M and anaplastic lymphoma kinase $(A L K)$ rearrangement (Ventana method).
Polymerase chain reaction showed no MET amplification. The patient discontinued afatinib and, in April 2014, initiated second-line treatment with pemetrexed and cisplatin chemotherapy for four cycles, and achieved a PFS of 8 months (Fig. 1). The patient had been treated with afatinib for a total of 40 months between December 2010 and April 2014, with no other anti-tumor therapy given during this time.

Further pleural re-biopsy in January 2015 identified EGFR Del19-positive, T790M-negative, and MET amplification- and ROS1 rearrangement-negative NSCLC (Fig. 1). At that time, next-generation sequencing (NGS) was not available for use in clinical practice, and the patient refused treatment with docetaxel. There were no further standard treatments for the patient, so from January 2015, the patient began treatment with osimertinib, which he obtained himself; it had not been approved in China at the time. Fortunately, he received benefit from osimertinib treatment and had PFS of approximately 19 months. Hepatic metastasis was detected in August 2016, and local ablation in the liver was carried out in August 2016 and again in November 2016. The patient developed aggravated cough and headache in November 2016, and leptomeningeal metastases were identified by brain magnetic resonance imaging (MRI). The patient received whole brain radiotherapy $(30 \mathrm{~Gy} / 10 \mathrm{~F})$ in December 2016, which was well tolerated. In January 2017, a lumbar puncture was performed and a sample of cerebrospinal fluid (CSF) was collected. NGS testing showed that CSF and blood plasma samples were both positive for EGFR T790M/C797S (cis) and Del19 mutations (Table 1). The patient tried treatment with osimertinib in combination with

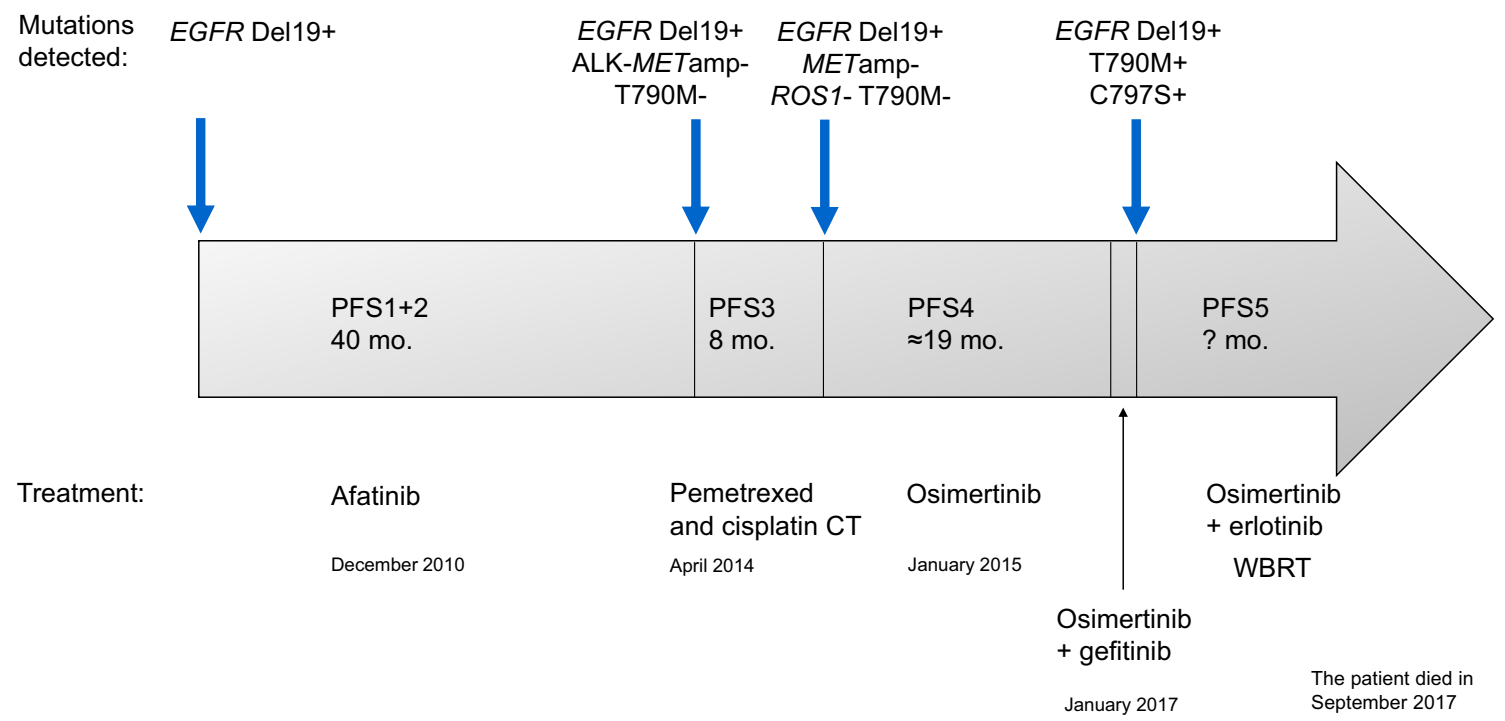

Fig. 1 Overview of medical history showing mutation types, treatments administered, and PFS outcomes. ALK anaplastic lymphoma kinase, $C T$ chemotherapy, Del19 deletions within exon 19, EGFR epidermal growth factor receptor gene, mo. months, PFS progressionfree survival, WBRT whole brain radiotherapy 

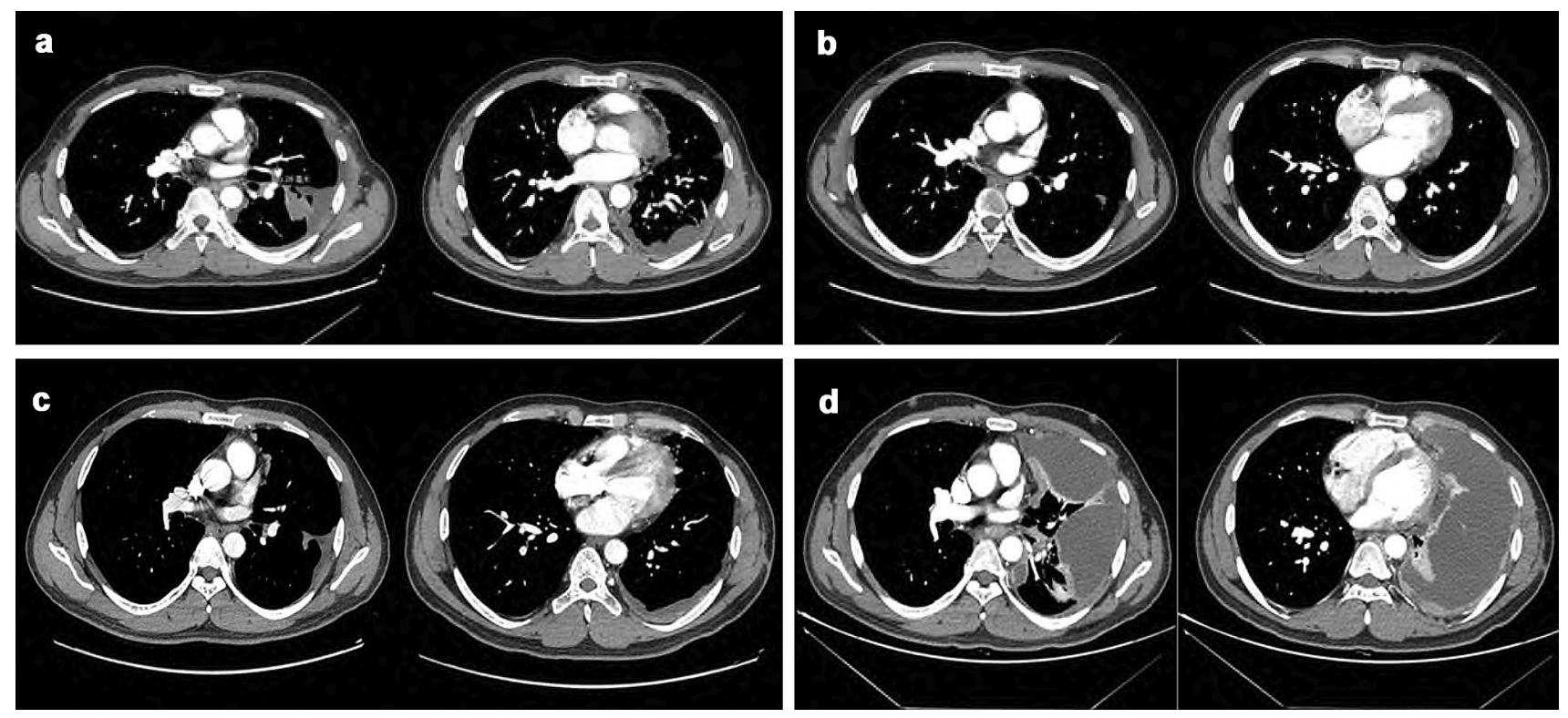

Fig. 2 Radiological assessment (CT scan) relating to first-line afatinib at a baseline (December 2010), b best response (March 2011), c slow progression (December 2013), and $\mathbf{d}$ full progression (April 2014). CT computed tomography

the first-generation EGFR TKIs gefitinib (250 mg daily) and erlotinib (150 mg daily) and received clinical benefit as his symptoms of cough, headache, and dizziness improved. At the last follow-up (May 2017), the patient was being treated with gefitinib, erlotinib and best supportive care; his general condition was still considered to be good, and symptoms of headache and dizziness had improved. The patient died in September 2017.

The case demonstrates long survival benefit from targeted treatment, especially the second-generation EGFR TKI afatinib. Although limited by detecting technology, the patient received the third-generation EGFR TKI, osimertinib, and also derived great benefit from the treatment.

This case demonstrates how appropriate sequencing of targeted therapy can result in long-term clinical benefit in patients with NSCLC. Initial identification of the EGFR Del19 mutation suggested that the cancer was driven by
EGFR activation, which led to initiation of targeted therapy with afatinib. PFS1 with first-line afatinib was 36 months, considerably longer than that typically reported in clinical trials of first-line afatinib (median PFS 11.0-11.1 months) [7, 20, 21]. This may reflect the absence of brain metastases at diagnosis and the fact that the patient had an initial objective response to treatment, both of which are associated with better prognosis during first-line afatinib treatment [22, 23]. Likewise, EGFR Del19 is associated with improved progression-free and overall survival in patients treated with afatinib rather than chemotherapy [24].

Following tumor progression, pleural biopsy analysis did not reveal the molecular mechanism of resistance to afatinib; at this time, the patient tested positive for EGFR Del19; EGFR T790M substitution, MET amplification and $A L K$ rearrangements were not observed. Nevertheless, uptake of repeated biopsies has been shown to help inform treatment

Table 1 Summary of genetic mutations in CSF and blood plasma samples (January 2017)

\begin{tabular}{|c|c|c|c|c|}
\hline Sample & Gene & Mutation type & Mutation result & Abundance (\%) \\
\hline \multirow[t]{3}{*}{ CSF } & $E G F R$ & Non-frameshift deletion mutation & $\begin{array}{l}\text { NM_005228.3(EGFR):c.2235_2249delGGAATT } \\
\text { AAGAGAAGC(p.Glu746_Ala750del) }\end{array}$ & 3.23 \\
\hline & $E G F R$ & Missense mutation & NM_005228.3(EGFR):c.2369C>T(p.Thr790Met) & 3.18 \\
\hline & $E G F R$ & Missense mutation & NM_005228.3(EGFR):c.2389T>A(p.Cys797Ser) & 3.11 \\
\hline \multirow[t]{3}{*}{ Blood plasma } & $E G F R$ & Missense mutation & NM_005228.3(EGFR):c.2369C>T(p.Thr790Met) & 39.10 \\
\hline & $E G F R$ & Non-frameshift deletion mutation & $\begin{array}{l}\text { NM_005228.3(EGFR):c.2235_2249delGGAATT } \\
\text { AAGAGAAGC(p.Glu746 Ala750del) }\end{array}$ & 30.86 \\
\hline & $E G F R$ & Missense mutation & NM_005228.3(EGFR):c.2389T>A(p.Cys797Ser) & 15.95 \\
\hline
\end{tabular}

$C S F$ cerebrospinal fluid, EGFR epidermal growth factor receptor gene 
decisions following acquired resistance to EGFR TKIs in other cases [25, 26]. Moreover, the continued effectiveness of EGFR-targeted therapies, namely osimertinib monotherapy and combination therapy, indicate that afatinib resistance may not have been EGFR-independent in this case. Despite the T790M-negative pleural biopsy, which could potentially have been caused by a false-negative EGFR test or an inadequate re-biopsied specimen for testing, the failure of afatinib treatment may still be attributable to T790M substitution, as intratumoral genetic heterogeneity, including T790M expression, has been documented [27-29]. A recent study has also suggested that primary EGFR Del19 mutation, brain metastasis, and longer PFS with initial EGFR TKI treatment are associated with acquired T790M resistance [30]. In this regard, analysis of both tissue biopsies and blood samples has been suggested to improve identification of the T790M mutation [25, 31]. This approach may allow more patients with T790M-positive disease to receive subsequent osimertinib after afatinib failure and potentially benefit from a longer chemotherapy-free period [25].

PFS following patient-initiated treatment with osimertinib was $\approx 19$ months, which is longer than that reported for second-line use of osimertinib in clinical trials (median PFS of 10.1 months) [32]. This may reflect disease metastasis being restricted largely to the intrathoracic cavity at the time of onset, without extrathoracic metastasis and, specifically, cerebral/brain metastasis, which has been associated with shorter PFS with second- and third-line osimertinib treatment in patients with NSCLC [33]. The total time on firstand second-line treatment was also longer than that observed in patients with Del19-positive tumors who received sequential first-line afatinib and second-line osimertinib in an observational, multicenter study (30.0 months) [34]. Consistent with that study, our findings provide further support for the sequential use of afatinib followed by osimertinib, particularly in Del19-positive NSCLC [34, 35].

After progression during osimertinib monotherapy, molecular typing revealed the mechanism of osimertinib resistance to be acquisition of the EGFR C797S substitution. Notably, this mutation was identified on the same allele as the T790M mutation (in cis), which is surprising given that previous studies have reported efficacy of first-/thirdgeneration EGFR TKI combinations against T790M/C797S mutations in trans but not in cis [36, 37].

Whilst this case exemplifies how long-term clinical benefit can be achieved through sequential use of TKIs, there is not yet a consensus on the optimal sequence of treatments $[5,18,19]$. Osimertinib was initially approved for secondline therapy of EGFR mutation-positive NSCLC following resistance to first- and second-generation TKIs [38]. However, following recent clinical evidence of improved PFS, CNS efficacy and tolerability compared with first-generation EGFR TKIs as first-line therapy [39], osimertinib has now been approved in this setting [40]. Whether osimertinib should be used as first-line treatment or reserved for secondline use remains a key question. In the absence of prospective trials specifically comparing sequential EGFR TKI regimens, the outcomes from comparative trials incorporating treatment crossover or assessing subsequent therapies will be important to inform the ongoing debate.

In this case, effective treatment sequencing led to a durable clinical benefit in a patient with EGFR mutation-positive stage IV NSCLC. PFS of 40 months was achieved with firstline afatinib, which was generally well tolerated, and subsequent osimertinib treatment afforded PFS of $\approx 19$ months, resulting in a combined PFS time of $\approx 60$ months with sequential afatinib and osimertinib. These findings provide further support for sequential use of afatinib followed by osimertinib in patients with EGFR mutation-positive NSCLC.

Acknowledgements Medical writing support for the development of this manuscript, under the direction of the authors, was provided by Victoria Steele and Caroline Perry, of Ashfield MedComms, an Ashfield Health company, and funded by Boehringer Ingelheim.

\section{Declarations}

Funding This work was supported by Boehringer Ingelheim. The LUX-Lung 6 study, in which the patient was enrolled while receiving treatment with afatinib, was funded by Boehringer Ingelheim. The authors received no direct compensation related to the development of the Manuscript. Editorial support was funded by Boehringer Ingelheim.

Conflict of interest H-YT, Y-SL, J-JY, H-JC, B-YJ, W-ZZ, and X-NY declare no potential conflicts of interest. Y-LW reports having received honoraria from AstraZeneca, Roche, BI, Pfizer, BMS, MSD, and Eli Lilly.

Author contributions H-YT designed the work, acquired, analyzed and interpreted the data, and drafted and/or substantively revised the manuscript. Y-SL drafted and/or substantively revised the manuscript. J-JY, H-JC, B-YJ, W-ZZ, and X-NY interpreted the data and drafted and/or substantively revised the manuscript. Y-LW designed the work, identified the case, interpreted the data, and drafted and/or substantively revised the manuscript. The authors were fully responsible for all content and editorial decisions, were involved at all stages of manuscript development and have approved the final version.

Ethics approval and consent to participate Not applicable.

Consent for publication Written informed consent for publication was obtained from the patient's family.

Availability of data and materials Data sharing is not applicable to this article as no datasets were generated or analyzed during the current study.

Open Access This article is licensed under a Creative Commons Attribution-NonCommercial 4.0 International License, which permits any non-commercial use, sharing, adaptation, distribution and reproduction in any medium or format, as long as you give appropriate credit to the 
original author(s) and the source, provide a link to the Creative Commons licence, and indicate if changes were made. The images or other third party material in this article are included in the article's Creative Commons licence, unless indicated otherwise in a credit line to the material. If material is not included in the article's Creative Commons licence and your intended use is not permitted by statutory regulation or exceeds the permitted use, you will need to obtain permission directly from the copyright holder. To view a copy of this licence, visit http://creativecommons.org/licenses/by-nc/4.0/.

\section{References}

1. Ferlay J, Soerjomataram I, Dikshit R, Eser S, Mathers C, Rebelo $\mathrm{M}$, et al. Cancer incidence and mortality worldwide: sources, methods and major patterns in GLOBOCAN 2012. Int J Cancer. 2015;136(5):E359-86.

2. Molina JR, Yang P, Cassivi SD, Schild SE, Adjei AA. Non-small cell lung cancer: epidemiology, risk factors, treatment, and survivorship. Mayo Clin Proc. 2008;83(5):584-94.

3. Nacht M, Dracheva T, Gao Y, Fujii T, Chen Y, Player A, et al. Molecular characteristics of non-small cell lung cancer. Proc Natl Acad Sci USA. 2001;98(26):15203-8.

4. Zer A, Leighl N. Promising targets and current clinical trials in metastatic non-squamous NSCLC. Front Oncol. 2014;4:329.

5. Girard N. Optimizing outcomes in EGFR mutation-positive NSCLC: which tyrosine kinase inhibitor and when? Future Oncol. 2018;14(11):1117-32.

6. Solca F, Dahl G, Zoephel A, Bader G, Sanderson M, Klein C, et al. Target binding properties and cellular activity of afatinib (BIBW 2992), an irreversible ErbB family blocker. J Pharmacol Exp Ther. 2012;343(2):342-50.

7. Sequist LV, Yang JC, Yamamoto N, O’Byrne K, Hirsh V, Mok T, et al. Phase III study of afatinib or cisplatin plus pemetrexed in patients with metastatic lung adenocarcinoma with EGFR mutations. J Clin Oncol. 2013;31(27):3327-34.

8. U.S. Food and Drug Administration. GILOTRIF ${ }^{\circledR}$ (afatinib). Highlights of Prescribing Information. https://www.accessdata.fda.gov/ drugsatfda_docs/label/2018/201292s014lbl.pdf. Accessed 24 Feb 2020.

9. Lim SM, Syn NL, Cho BC, Soo RA. Acquired resistance to EGFR targeted therapy in non-small cell lung cancer: mechanisms and therapeutic strategies. Cancer Treat Rev. 2018;65:1-10.

10. Neel DS, Bivona TG. Resistance is futile: overcoming resistance to targeted therapies in lung adenocarcinoma. NPJ Precis Oncol. 2017;1:3.

11. Sequist LV, Waltman BA, Dias-Santagata D, Digumarthy S, Turke $\mathrm{AB}$, Fidias $\mathrm{P}$, et al. Genotypic and histological evolution of lung cancers acquiring resistance to EGFR inhibitors. Sci Transl Med. 2011;3(75):75ra26.

12. Yu HA, Arcila ME, Rekhtman N, Sima CS, Zakowski MF, Pao $\mathrm{W}$, et al. Analysis of tumor specimens at the time of acquired resistance to EGFR-TKI therapy in 155 patients with EGFRmutant lung cancers. Clin Cancer Res. 2013;19(8):2240-7.

13. Jenkins S, Chih-Hsin Yang J, Janne PA, Thress KS, Yu K, Hodge R, et al. EGFR mutation analysis for prospective patient selection in two phase II registration studies of osimertinib. J Thorac Oncol. 2017;12(8):1247-56.

14. Pao W, Miller VA, Politi KA, Riely GJ, Somwar R, Zakowski $\mathrm{MF}$, et al. Acquired resistance of lung adenocarcinomas to gefitinib or erlotinib is associated with a second mutation in the EGFR kinase domain. PLoS Med. 2005;2(3):e73.

15. Yun $\mathrm{CH}$, Mengwasser KE, Toms AV, Woo MS, Greulich H, Wong KK, et al. The T790M mutation in EGFR kinase causes drug resistance by increasing the affinity for ATP. Proc Natl Acad Sci USA. 2008;105(6):2070-5.

16. Yoshikawa S, Kukimoto-Niino M, Parker L, Handa N, Terada T, Fujimoto T, et al. Structural basis for the altered drug sensitivities of non-small cell lung cancer-associated mutants of human epidermal growth factor receptor. Oncogene. 2013;32(1):27-38.

17. Cross DA, Ashton SE, Ghiorghiu S, Eberlein C, Nebhan CA, Spitzler PJ, et al. AZD9291, an irreversible EGFR TKI, overcomes T790M-mediated resistance to EGFR inhibitors in lung cancer. Cancer Discov. 2014;4(9):1046-61.

18. Recondo G, Facchinetti F, Olaussen KA, Besse B, Friboulet L. Making the first move in EGFR-driven or ALK-driven NSCLC: first-generation or next-generation TKI? Nat Rev Clin Oncol. 2018;15(11):694-708.

19. Hirsh V. Turning EGFR mutation-positive non-small-cell lung cancer into a chronic disease: optimal sequential therapy with EGFR tyrosine kinase inhibitors. Ther Adv Med Oncol. 2018;10:1758834017753338.

20. Wu YL, Zhou C, Hu CP, Feng J, Lu S, Huang Y, et al. Afatinib versus cisplatin plus gemcitabine for first-line treatment of Asian patients with advanced non-small-cell lung cancer harbouring EGFR mutations (LUX-Lung 6): an open-label, randomised phase 3 trial. Lancet Oncol. 2014;15(2):213-22.

21. Park K, Tan EH, O’Byrne K, Zhang L, Boyer M, Mok T, et al. Afatinib versus gefitinib as first-line treatment of patients with EGFR mutation-positive non-small-cell lung cancer (LUX-Lung 7): a phase $2 \mathrm{~B}$, open-label, randomised controlled trial. Lancet Oncol. 2016;17(5):577-89.

22. Liang SK, Hsieh MS, Lee MR, Keng LT, Ko JC, Shih JY. Realworld experience of afatinib as a first-line therapy for advanced EGFR mutation-positive lung adenocarcinoma. Oncotarget. 2017;8(52):90430-43.

23. Liang SK, Lee MR, Liao WY, Ho CC, Ko JC, Shih JY. Prognostic factors of afatinib as a first-line therapy for advanced EGFR mutation-positive lung adenocarcinoma: a real-world, large cohort study. Oncotarget. 2018;9(34):23749-60.

24. Kuan FC, Kuo LT, Chen MC, Yang CT, Shi CS, Teng D, et al. Overall survival benefits of first-line EGFR tyrosine kinase inhibitors in EGFR-mutated non-small-cell lung cancers: a systematic review and meta-analysis. Br J Cancer. 2015;113(10):1519-28.

25. Hochmair MJ, Buder A, Schwab S, Burghuber OC, Prosch H, Hilbe W, et al. Liquid-biopsy-based identification of EGFR T790M mutation-mediated resistance to afatinib treatment in patients with advanced EGFR mutation-positive NSCLC, and subsequent response to osimertinib. Target Oncol. 2019;14:75-83.

26. Buder A, Hochmair MJ, Schwab S, Bundalo T, Schenk P, Errhalt $\mathrm{P}$, et al. Cell-free plasma DNA-guided treatment with osimertinib in patients with advanced EGFR-mutated NSCLC. J Thorac Oncol. 2018;13(6):821-30.

27. Gerlinger M, Rowan AJ, Horswell S, Math M, Larkin J, Endesfelder D, et al. Intratumor heterogeneity and branched evolution revealed by multiregion sequencing. $\mathrm{N}$ Engl J Med. 2012;366(10):883-92.

28. Inukai M, Toyooka S, Ito S, Asano H, Ichihara S, Soh J, et al. Presence of epidermal growth factor receptor gene T790M mutation as a minor clone in non-small cell lung cancer. Cancer Res. 2006;66(16):7854-8.

29. Piotrowska Z, Hazar-Rethinam M, Rizzo C, Nadres B, Van Seventer EE, Shahzade HA, et al. Heterogeneity and coexistence of T790M and T790 wild-type resistant subclones drive mixed response to third-generation epidermal growth factor receptor inhibitors in lung cancer. JCO Precis Oncol. 2018;2018:PO.17.00263.

30. Wei B, Zhao C, Li J, Zhao J, Ren P, Yang K, et al. Combined plasma and tissue genotyping of EGFR T790M benefits 
NSCLC patients: a real-world clinical example. Mol Oncol. 2019;13(5):1226-34.

31. Normanno N, Maiello MR, Chicchinelli N, Iannaccone A, Esposito C, De Cecio R, et al. Targeting the EGFR T790M mutation in non-small-cell lung cancer. Expert Opin Ther Targets. 2017;21(2):159-65.

32. Mok TS, Wu YL, Ahn MJ, Garassino MC, Kim HR, Ramalingam SS, et al. Osimertinib or platinum-pemetrexed in EGFR T790Mpositive lung cancer. N Engl J Med. 2017;376(7):629-40.

33. Auliac JB, Perol M, Planchard D, Monnet I, Wislez M, Doubre H, et al. Real-life efficacy of osimertinib in pretreated patients with advanced non-small cell lung cancer harboring EGFR T790M mutation. Lung Cancer. 2019;127:96-102.

34. Hochmair MJ, Morabito A, Hao D, Yang C-T, Soo RA, Yang JC-H, et al. Sequential afatinib and osimertinib in patients with EGFR mutation-positive non-small-cell lung cancer: final analysis of the GioTag study. Future Oncol 2020;16(34):2799-808.

35. Hochmair MJ, Morabito A, Hao D, Yang CT, Soo RA, Yang $\mathrm{JC}$, et al. Sequential afatinib and osimertinib in patients with EGFR mutation-positive non-small-cell lung cancer: updated analysis of the observational GioTag study. Future Oncol. 2019;15(25):2905-14.
36. Wang Z, Yang JJ, Huang J, Ye JY, Zhang XC, Tu HY, et al. Lung adenocarcinoma harboring EGFR T790M and in trans C797S responds to combination therapy of first- and third-generation EGFR TKIs and shifts allelic configuration at resistance. J Thorac Oncol. 2017;12(11):1723-7.

37. Niederst MJ, Hu H, Mulvey HE, Lockerman EL, Garcia AR, Piotrowska Z, et al. The allelic context of the C797S mutation acquired upon treatment with third-generation EGFR inhibitors impacts sensitivity to subsequent treatment strategies. Clin Cancer Res. 2015;21(17):3924-33.

38. Greig SL. Osimertinib: first global approval. Drugs. 2016;76(2):263-73

39. Soria JC, Ohe Y, Vansteenkiste J, Reungwetwattana T, Chewaskulyong $\mathrm{B}$, Lee $\mathrm{KH}$, et al. Osimertinib in untreated EGFRmutated advanced non-small-cell lung cancer. N Engl J Med. 2018;378(2):113-25.

40. U.S. Food and Drug Administration. TAGRISSO ${ }^{\mathrm{TM}}$ (osimertinib). Highlights of Prescribing Information. https://www.accessdata. fda.gov/drugsatfda_docs/label/2015/208065s000lbl.pdf. Accessed 24 Feb 2020. 